\title{
Protection Efficacy of the rLTB-R1 Chimera against Experimental Swine Mycoplasmal Pneumonia
}

\author{
Marcos Roberto Alves Ferreira', Paula Fonseca Finger ${ }^{2}$, Carolina Georg Magalhães ${ }^{3}$, \\ Carlos Eduardo Pouey da Cunha', Clóvis Moreira Júnior', Jalusa Deon Kich4, \\ Nelson Morés ${ }^{4}$ Ângela Nunes Moreira ${ }^{1,5}$, Odir Antônio Dellagostin' \& Fabricio Rochedo Conceição'
}

\begin{abstract}
Background: Mycoplasma hyopneumoniae is the etiological agent of the Swine Mycoplasmal Pneumonia (SMP), one of the most economically significant diseases in the swine industry worldwide. Commonly used vaccines for SMP control consist of inactivated whole cells (bacterins). These vaccines are efficacious against $M$. hyopneumoniae challenge, but do not prevent colonization by the pathogen or completely eliminate pneumonia. P97 adhesin is conserved in the M. pneumoniae virulent strains, therefore it is an attractive target to be used in recombinant vaccines against $M$. hyopneumoniae. The aim of the present study was to evaluate protection afforded by rLTB-R1, a recombinant chimera composed by LTB fused with the R1 repeat region of P97 adhesin of M. hyopneumoniae, in specific-pathogen-free (SPF) piglets vaccinated by intranasal or intramuscular route and challenged with a pathogenic strain of $M$. hyopneumoniae.
\end{abstract}

Materials, Methods \& Results: PCR products of the LTB and R1 coding sequences were fused, then cloned into pETDEST42 ${ }^{\mathrm{TM}}$ expression vector. The rLTB-R1 was expressed in Escherichia coli BL21 (DE3) Salt induction (SI). The piglets were divided into three groups: four piglets were intranasally vaccinated with $1 \mathrm{mg}$ of rLTB-R1 solubilized in $1 \mathrm{~mL}$ of PBS at 0 and 14 days (IN rLTB-R1 group); four piglets were intramuscularly vaccinated with $1 \mathrm{mg}$ of rLTB-R1 solubilized in 1 $\mathrm{mL}$ of PBS at 0 and 14 days (IM rLTB-R1 group); three piglets were intranasally and intramuscularly inoculated with $1 \mathrm{~mL}$ of PBS (control group). Two weeks after the last immunization (28 day), piglets were intratracheally challenged with $10 \mathrm{~mL}$ of a suspension containing $10^{9}$ color-changing unit (CCU) of pathogenic M. hyopneumoniae 7448 strain on three consecutive days. Until the challenge (28 days), intranasal and intramuscular vaccination with rLTB-R1 induced seroconversions of antiR1 systemic antibodies of 1.6 and $4.6 \times$, respectively. The IN rLTB-R1 group had no pulmonary lesion, rLTB-R1 conferred protection against experimental SMP. On the other hand, IM rLTB-R1 and control groups had on average $7.24 \%$ and $8.46 \%$ of pulmonary lesion, respectively, showing that intramuscular vaccination with rLTB-R1 did not confer protection.

Discussion: The rLTB-R1, when intranasally administrated to mice, elicited production of anti-R1 IgA in trachea and bronchi as well as specific Th1 response, suggesting an adequate stimulation of the mucosal immune system. We believe that rLTB-R1 induced a similar immune response in piglets intranasally vaccinated, conferring protection against experimental SMP. The present study, the rLTB-R1 alone, without any chemical adjuvant, stimulated a significant seroconversion of anti-R1 systemic antibodies in pigs intramuscularly vaccinated, showing the potential of LTB as a parenteral adjuvant in swine vaccination. Previous work has shown that the intramuscular administration route was evaluated in pigs because mice intramuscularly vaccinated with rLTB-R1 presented significant levels of anti-R1 IgA in trachea and bronchi, suggesting that rLTB can stimulate some degree of mucosal immunity even if not delivered by a mucosal route. However, in the present study, piglets intramuscularly vaccinated with rLTB-R1 presented high levels of anti-R1 systemic antibodies, they were not protected. On the other hand, intranasal vaccination of piglets with rLTB-R1 elicited low levels of antiR1 systemic antibodies $(1.6 \times$ at 28 days), but it conferred full protection against experimental SMP. The present study demonstrated that intranasal vaccination of piglets with rLTB-R1 conferred protection against experimental SMP. A more detailed analysis of the protective immune response induced by rLTB-R1 in pigs is currently being performed.

Keywords: Mycoplasma hyopneumoniae, Swine Mycoplasmal Pneumonia, mucosal vaccination, P97 adhesin, LTB.

DOI: $10.22456 / 1679-9216.90862$

${ }^{1}$ Centro de Desenvolvimento Tecnológico (CDTec) \& ${ }^{5}$ Faculdade de Nutrição, Universidade Federal de Pelotas (UFPel), Pelotas, RS, Brazil. ${ }^{2}$ Medicina Veterinária, Universidade Federal do Pampa (Unipampa), Uruguaiana, RS. ${ }^{3}$ Instituto Butantan, Universidade de São Paulo (USP), São Paulo, SP, Brazil. ${ }^{4}$ Empresa Brasileira de Pesquisa Agropecuária (EMBRAPA), Centro Nacional de Pesquisa de Suínos e Aves (CNPSA), Concórdia, SC, Brazil. CORRESPONDENCE: M.R.A. Ferreira [marcosferreiravet@gmail.com - Tel: +55 (53) 32757583]. Centro de Desenvolvimento Tecnológico (CDTec), Universidade Federal de Pelotas (UFPel), Campus Universitário Capão do Leão. CEP 96160-000 Pelotas, RS, Brazil. 


\section{INTRODUCTION}

Mycoplasma hyopneumoniae is the etiological agent of the Swine Mycoplasmal Pneumonia (SMP), one of the most economically significant diseases in the swine industry worldwide. Adherence of M. hyopneumoniae to the swine respiratory epithelial cells causes reduction of ciliary activity, ciliostasis and loss of cilia [3,22,24-26].

Adherence of $M$. hyopneumoniae to the swine cilia is mediated by $\mathrm{R} 1$ repeat region (AAKPV-E) of the P97 adhesin [12], which has been shown to be highly immunogenic for pigs [17,19]. P97 adhesin is conserved in the M. pneumoniae virulent strains, therefore it is an attractive target to be used in recombinant vaccines against $M$. hyopneumoniae [28]. However, King et al. [9] observed that recombinant P97 did not protect swine from becoming infected with $M$. hyopneumoniae nor reduced the severity of the disease as measured by lung lesions. It is conceivable that failure in protection was due to the route of vaccination used (intramuscular), since parenteral administration of immunogens does not induce an adequate mucosal immunity [29]. An alternative to this problem is the utilization of mucosal adjuvants. B subunit of Escherichia coli heat-labile enterotoxin (LTB) has been shown to be a potent mucosal adjuvant, stimulating a strong systemic and secretory response of antibodies against co-administrated or coupled antigens $[2,7,13,15]$.

The aim of the present study was to evaluate protection afforded by rLTB-R1, a recombinant chimera composed by LTB fused with the $\mathrm{R} 1$ repeat region of P97 adhesin of M. hyopneumoniae, in specificpathogen-free (SPF) piglets vaccinated by intranasal or intramuscular route and challenged with a pathogenic strain of M. hyopneumoniae.

\section{MATERIALS AND METHODS}

Cloning, expression, and purification of recombinant ETX

The rLTB-R1 was constructed, expressed and characterized as previously described [7]. Briefly, PCR products of the LTB and R1 coding sequences were fused, then cloned into $\mathrm{pENTR/SD}$ cloning plasmid ${ }^{1}$. The fragment was then transferred by site specific recombination to $\mathrm{pETDEST} 42^{\mathrm{TM}}$ expression vector ${ }^{1}$, which allows fusion of the protein with a $\mathrm{C}$ terminal $6 \times$ His tag. After transformation of the construct into an appropriate $E$. coli BL21 (DE3) SI, and induction with $\mathrm{NaCl}$ and isopropyl $\beta$-D-1-thiogalactopyranoside (IPTG), the recombinant protein was purified by Ni-NTA affinity chromatography, quantified by the Bradford method and characterized by Western blot.

\section{Bacterial strain}

The pathogenic Mycoplasma hyopneumoniae 7448 homologous strain was used in the challenge experiment. This strain was grown in Friis medium ${ }^{2}$. Cultures consisted of $125 \mathrm{~mL}$ of Friis medium in Erlenmeyer flasks incubated at $37^{\circ} \mathrm{C}$ with slow agitation until the culture reached mid-log phase, as indicated by color change and turbidity.

\section{Experimental design}

Eleven 6 to 8-week-old SPF piglets were obtained from Embrapa Suínos e Aves, Concórdia, SC, Brazil. These animals had no serological evidence of exposure to $M$. hyopneumoniae as examined by an ELISA using Tween 20 soluble proteins of $M$. hyopneumoniae as antigens $[16,18]$. They were allocated into three groups: four piglets were intranasally vaccinated with $1 \mathrm{mg}$ of rLTB-R1 solubilized in $1 \mathrm{~mL}$ of PBS at 0 and 14 days (IN rLTB-R1 group); four piglets were intramuscularly vaccinated with $1 \mathrm{mg}$ of rLTB$\mathrm{R} 1$ solubilized in $1 \mathrm{~mL}$ of PBS at 0 and 14 days (IM rLTB-R1 group) and; three piglets were intranasally and intramuscularly inoculated with $1 \mathrm{~mL}$ of PBS (control group). Two weeks after the last immunization (28 day), piglets were intratracheally challenged with $10 \mathrm{~mL}$ of a suspension containing $10^{9}$ color-changing unit (CCU) of pathogenic M. hyopneumoniae 7448 strain on three consecutive days. Food and water were provided ad libitum. Eight weeks after the challenge exposure, the piglets were euthanatized and necropsied. For determination of the extent of gross lung lesions, the percentage of lung affected by pneumonia was calculated following Morrison et al. [14]. Lung samples were microscopically examined for characteristic mycoplasmal pneumonia histopathological damage as previously described [25]. Slides with lung tissue (3-5 $\mu \mathrm{m}$ thick) were also used for immunohistochemistry using polyclonal sera against $M$. hyopneumoniae, produced by Embrapa Suínos e Aves, and Vectastain ABC $\mathrm{kit}^{\circledR 3}$. Briefly, paraffin was removed from the slides and tissue rehydrated. Antigen was recovered with citrate buffer $(\mathrm{pH} \mathrm{6,0)}$ and heat (microwave oven for $5 \mathrm{~min}, 700 \mathrm{~W}$ ) and enzymatically digested with pepsin $(0.04 \%$ in $\mathrm{HCl} 0.001 \mathrm{~N}-\mathrm{pH} 7.8)$ for $10 \mathrm{~min}$ at $37^{\circ} \mathrm{C}$. 
Endogenous peroxidase was inactivated with hydrogen peroxide (3\%) for $5 \mathrm{~min}$. Slides were incubated with the primary antibody for $2 \mathrm{~h}$ at $37^{\circ} \mathrm{C}$, followed by binding to the Vectastain $\mathrm{ABC} \mathrm{kit}^{\circledR 3}$. Reaction was developed with 3-amino-9-ethylcarbazole (AEC) for 5 $\min$ at $37^{\circ} \mathrm{C}$. Mayer's hematoxylin was used to conter stain the slides. Slides were washed for $5 \mathrm{~min}$ between each step with PBS (pH 7.4) plus Tween 20 (0.05\%). Slides were read with an optic microscope.

Sample sera were weekly collected. Seroconversions of anti-R1 systemic antibodies determined by ELISA and Western blot analysis were performed as previously described [7]. Mean seroconversion of anti-R1 systemic antibodies, pulmonary lesion scores and immunohistochemistry scores were subjected to analysis of variance. The following criteria were used in statistical analysis of pulmonary lesions and immunohistochemistry, respectively: score 1 for presence of pulmonary lesion and 0 for absence; imunohistocemestry score 0 : absence of staining; weak ( 1 cross), moderate ( 2 crosses), and strong ( 3 crosses) staining in ciliated epithelium of bronchia and bronchioles [20]. The Student's $t$-test was used to determine significant differences $(P<0.05)$ among the means of each group through the Statistix software.

\section{RESULTS}

The IN rLTB-R1 group had no pulmonary lesion, therefore intranasal vaccination with rLTB-R1 conferred full protection against experimental SMP $(P$ $<0.001$ in comparison with IM rLTB-R1 and control groups). On the other hand, IM rLTB-R1 and control groups had on average $7.24 \%$ and $8.46 \%$ of pulmonary lesion, respectively, showing that intramuscular vaccination with rLTB-R1 did not confer protection. Immunohistochemistry assay showed that none of the piglets of the IN rLTB-R1 group had evidence of infection $(P<0.001$ in comparison with IM rLTB-R1 and control groups), while all animals of the IM rLTB-R1 and control groups were positive (Figure 1). Table 1 shows the results of the challenge test.

Mean seroconversion of anti-R1 systemic antibodies is shown in Figure 2. Both intranasal and intramuscular vaccination with rLTB-R1 induced significant seroconversions $(P<0.05)$ at 14 and 28 days in comparison with the control group, showing that the recombinant chimera was immunogenic to pigs. Until the challenge (28 days), intranasal and intramuscular vaccination with rLTB-R1 induced seroconversions of anti-R1 systemic antibodies of 1.6 and $4.6 \times$, respectively. Four weeks after the challenge (56 days), seroconversions of anti-R1 systemic antibodies of all groups increased, suggesting immunologic stimulation by the challenge strain. Western blot analysis showed that the pooled sera from piglets intramuscularly vaccinated with rLTB-R1 recognized the $\mathrm{P} 97$ adhesin of the $M$. hyopneumoniae 7448 pathogenic strain and the products derived from its proteolytic processing (data not shown).

Table 1. Lesion score and amount of antigen by immunohistochemistry (IHC) in bronchioles of different treatment groups after challenge with pathogenic Mycoplasma hypneumoniae strain 7448.

\begin{tabular}{ccc}
\hline Groups & \% Lesions & Immunohistochemistry \\
\hline IM rLTB-R1 & 3.12 & +++ \\
\hline Piglet 1 & 23.87 & +++ \\
Piglet 2 & 1.0 & ++ \\
Piglet 3 & 1.0 & + \\
Piglet 4 & 7.24 & - \\
Mean & & - \\
\hline IN rLTB-R1 & 0 & - \\
\hline Piglet 1 & 0 & - \\
Piglet 2 & 0 & \\
Piglet 3 & 0 & + \\
Piglet 4 & 0 & +++ \\
Mean & & + \\
Control & 1.0 & + \\
\hline Piglet 1 & 23.37 & \\
Piglet 2 & 1.0 & \\
Piglet 3 & 8.46 & \\
Mean & &
\end{tabular}




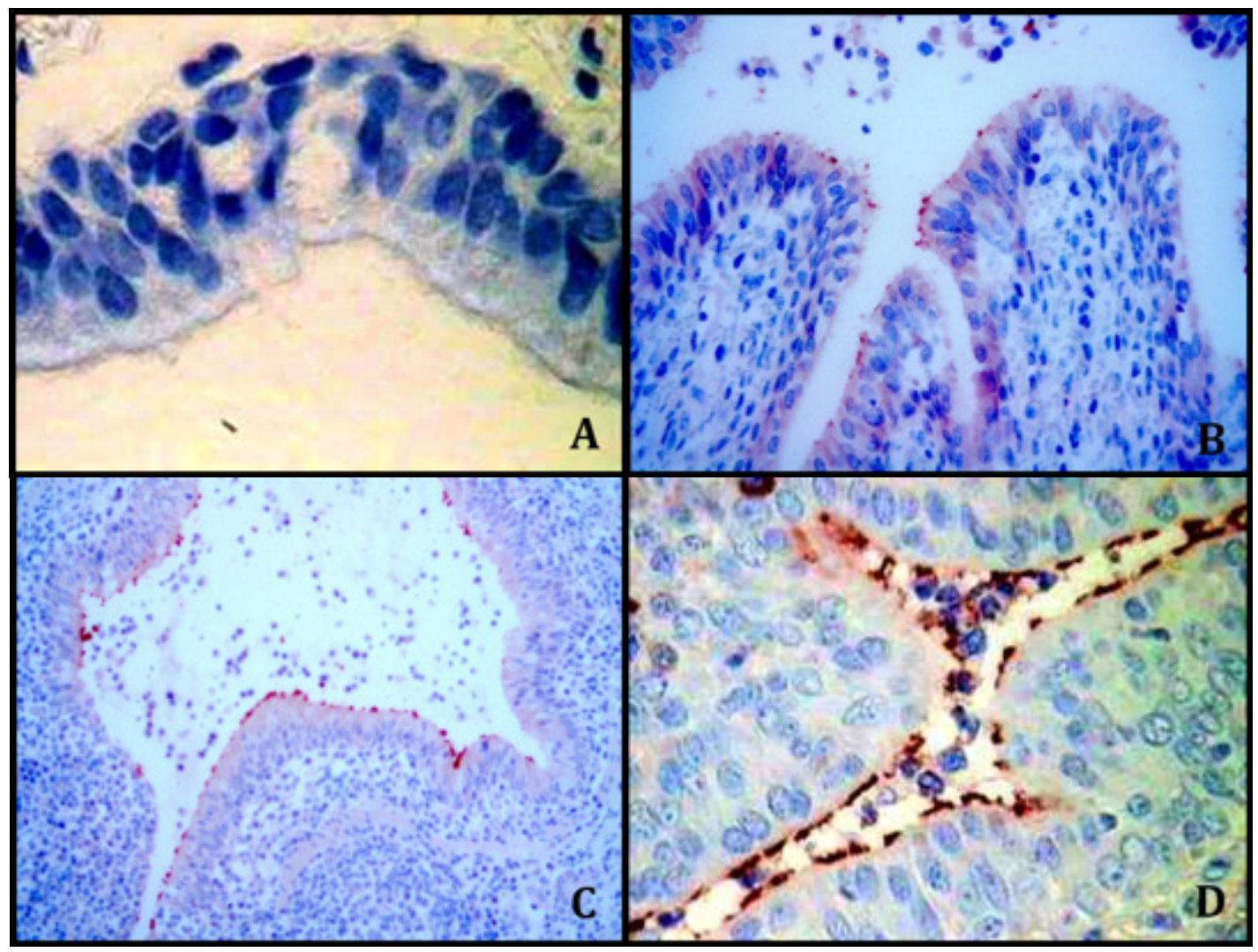

Figure 1. Immunohistochemistry scores in bronchioles of animals challenged with Mycoplasma hyopneumoniae strain 7448. A- Absence of staining in cilia of epithelial cells [100x]. B- Positive staining (+) in cilia of epithelial cells [50x]. C- Positive staining (++) in cilia of epithelial cells [40x]. D- Positive staining (+++) in cilia of epithelial cells [100x]. Positive staining is represented by red colour.

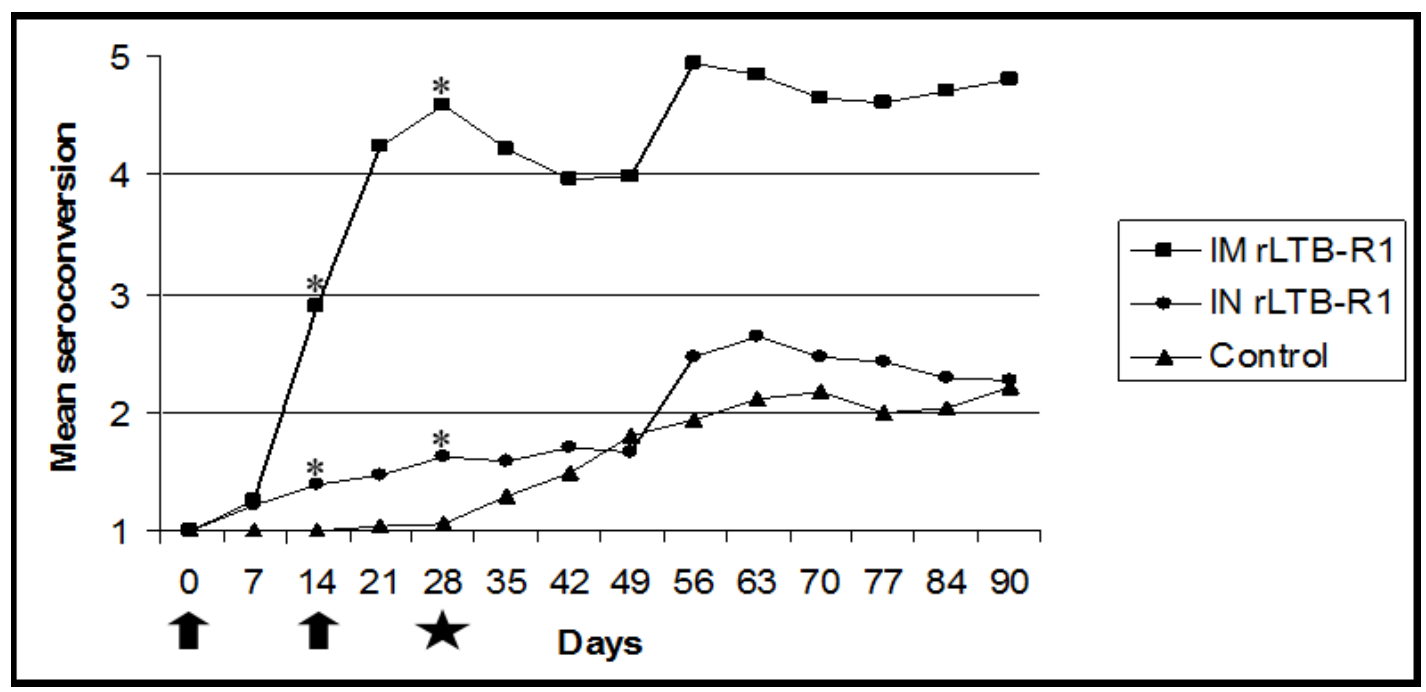

Figure 2. Mean seroconversion of anti-R1 systemic antibodies determined by ELISA using rR1 as antigen. Piglets were intranasally or intramuscularly vaccinated with $1 \mathrm{mg}$ of rLTB-R 1 at 0 and 14 days (arrows). Two weeks after the last immunization, piglets were intratracheally challenged with $10^{9} \mathrm{CCU}$ of pathogenic Mycoplasma hyopneumoniae 7448 strain. $* P<0.05$ in comparison to the control group.

\section{DISCUSSION}

In the present study we evaluated protection induced by the rLTB-R1 chimera, a new vaccine candidate for controlling SMP composed by the B subunit of $E$. coli heat-labile enterotoxin fused with the R1 repeat region of the $\mathrm{P} 97$ adhesin of $M$. hyopneumoniae. Commonly used vaccines to SMP control consist of inactivated whole cell preparations. These vaccines 
can provide protection for pigs against experimental M. hyopneumoniae challenge but cannot completely eliminate pneumonia or significantly reduce colonization by the microorganism. Inactivated whole cell vaccines are efficacious in the prevention of infectious diseases controlled by systemic antibodies; however, are incapable of inducing a suitable mucosal immunity $[5,15]$.

The rLTB-R1, when intranasally administrated to mice, elicited production of anti-R1 IgA in trachea and bronchi as well as specific Th1 response [7], suggesting an adequate stimulation of the mucosal immune system. We believe that rLTB-R1 induced a similar immune response in piglets intranasally vaccinated, conferring protection against experimental SMP. Several researchers suggested that mucosal $(\operatorname{IgA})$ and cellular immune responses may be important in the resolution of $M$. hyopneumoniae infection $[6,7,11,18]$. Although the evaluation of the humoral and/or cellular immune responses induced by an experimental vaccine is of interest, the most important parameter to be evaluated is the level of protection afforded by the vaccine candidate.

To stimulate a significant systemic IgG response against the R1 region of the P97 adhesin of $M$. hyopneumoniae in pigs, the PE( $\triangle \mathrm{III})-\mathrm{RR} 1$ recombinant chimera, composed by Pseudomonas exotoxin fused with the R1 repeat region of the P97 adhesin of M. hyopneumoniae, needed to be adjuvanted with aluminum hydroxide and co-administrated with a commercial bacterin against SMP [4]. Galli et al. [8] evaluated five M. hyopneumoniae antigens (P37, $\mathrm{P} 42, \mathrm{P} 46$ e $\mathrm{P} 95)$ as vaccine in mice, of which, P42, P95 and pcDNA3/ P46 were effective of generating immune response. DNA vaccine pcDNA3/P46 induced antibodies IgG1 e IgG2a, as well as higher levels of INF $\gamma$ compared with subunit vaccines. Studies by Chen et al. [6] using DNA vaccines containing antigens $\mathrm{P} 36, \mathrm{P} 46, \mathrm{NrdF}, \mathrm{P} 97$ or P97R1, antigen P46 generated mainly IgG. The present study, the rLTB-R1 alone, without any chemical adjuvant, stimulated a significant seroconversion of anti-R1 systemic antibodies in pigs intramuscularly vaccinated, showing the potential of LTB as a parenteral adjuvant in swine vaccination.

The intramuscular administration route was evaluated in pigs because mice intramuscularly vaccinated with rLTB-R1 presented significant levels of anti-R1 IgA in trachea and bronchi [7], suggesting that rLTB can stimulate some degree of mucosal immunity even if not delivered by a mucosal route. Similar results were obtained [27] using rLTB as adjuvant and urease of Helicobacter pylori as immunogen. Although piglets intramuscularly vaccinated with rLTB-R1 presented high levels of anti-R1 systemic antibodies, they were not protected. Similarly, intramuscular vaccination of piglets with recombinant GST-P97 using complete Freund's adjuvant elicited a strong systemic humoral response, but it was incapable of protecting against experimental SMP [9]. On the other hand, intranasal vaccination of piglets with rLTB-R1 elicited low levels of anti-R1 systemic antibodies ( $1.6 \times$ at 28 days), but it conferred full protection against experimental SMP. Other reports showed that serum antibodies induced by commercial M. hyopneumoniae vaccines do not correlate with protection against $M$. hyopneumoniae challenge [10,23]. Therefore, serum antibodies may not play a protective role in SMP, suggesting that an adequate stimulation of the mucosal immune system is important in SMP prevention. In human and chickens, a positive correlation between anti-Mycoplasma specific IgA levels and protection has been established [1,21].

Recombinant vaccines using P97 adhesin as antigen have been evaluated. Ogawa et al. [17] demonstrated Erysipelothrix rhusiopathiae 65-0.15 strain expressing on the surface the C-terminal portion of M. hyopneumoniae $\mathrm{P} 97$ adhesin, orally administrated to piglets, reduced the severity of pneumonic lung lesions caused by experimental SMP. It was demonstrated that mice immunized orally with attenuated Salmonella typhimurium aroA strain CS332 harboring a eukaryotic or prokaryotic expression vector encoding P97 adhesin did not produce a local and systemic humoral response against P97 [5]. Okamba et al. [18] showed a significant anti-P97 immune response in mice intranasally vaccinated with a replicationdefective recombinant adenovirus expressing the C-terminal portion of P97 adhesin. The vaccine candidates against SMP previously mentioned use attenuated pathogens as vectors, a strategy whose tendency is to disappear.

The present study demonstrated that intranasal vaccination of piglets with rLTB-R1 conferred protection against experimental SMP. A more detailed analysis of the protective immune response induced by rLTB-R1 in pigs is currently being performed. 


\section{MANUFACTURERS}

${ }^{1}$ Invitrogen Corporation. Carlsbad, CA, USA.

${ }^{2}$ HiMedia Laboratories Pvt. Munbai, India.

${ }^{3}$ Vector Laboratories Inc. Burlingame, CA, USA.

Ethical approval. Animal experiments were carried out according to the guidelines of the Ethics Committee in Animal Experimentation of the Universidade Federal de Pelotas (approval number 5614).
Acknowledgments. This work was supported by grants from CNPq. F.R.C was supported by a scholarship from CAPES. We are grateful to Marni Fracasso Ramenzoni, Remídio Vizzotto and Altair Althaus from Embrapa Suínos e Aves for technical assistance.

Declaration of interest. The authors report no conflicts of interest. The authors alone are responsible for the content and writing of the paper

\section{REFERENCES}

1 Avakian A.P. \& Ley D.H. 1993. Protective immune response to Mycoplasma gallisepticum demonstrated in respiratorytract washings from M. gallisepticum-infected chickens. Avian Diseases. 37(3): 697-705.

2 Barate A.K., Cho Y., Truong Q.L. \& Hahn T.W. 2014. Immunogenicity of IMS 1113 plus soluble subunit and chimeric proteins containing Mycoplasma hyopneumoniae P97 C-terminal repeat regions. FEMS Microbiology Letters. 352(2): 213-220.

3 Blanchard B., Vena M.M., Cavalier A., Le Lannic J., Gouranton J. \& Kobisch M. 1992. Electron microscopic observation of the respiratory tract of SPF piglets inoculated with Mycoplasma hyopneumoniae. Veterinary Microbiology. 30(4): 329-341.

4 Chen J.R., Liao C.W., Mao S.J. \& Weng C.N. 2001. A recombinant chimera composed of repeat region RR1 of Mycoplasma hyopneumoniae adhesin with Pseudomonas exotoxin: in vivo evaluation of specific IgG response in mice and pigs. Veterinary Microbiology. 80(4): 347-357.

5 Chen A.Y., Fry S.R., Forbes-Faulkner J., Daggard G. \& Mukkur T.K. 2006. Evaluation of the immunogenicity of the P97R1 adhesin of Mycoplasma hyopneumoniae as a mucosal vaccine in mice. Journal of Medical Microbiology. 55(7): 923-929.

6 Chen A.Y., Fry S.R., Daggard G.E. \& Mukkur T.K. 2008. Evaluation of immune response to recombinant potential protective antigens of Mycoplasma hyopneumoniae delivered as cocktail DNA and/or recombinant protein vaccines in mice. Vaccine. 26(34): 4372-8.

7 Conceição F.R., Moreira A.N. \& Dellagostin O.A. 2006. A recombinant chimera composed of R1 repeat region of Mycoplasma hyopneumoniae P97 adhesin with Escherichia coli heat-labile enterotoxin B subunit elicits immune response in mice. Vaccine. 24(29-30): 5734-5743.

8 Galli V., Simionatto S., Marchioro S.B., Fisch A., Gomes C.K., Conceicão F.R. \& Dellagostin O.A. 2012. Immunisation of mice with Mycoplasma hyopneumoniae antigens P37, P42, P46 and P95 delivered as recombinant subunit or DNA vaccines. Vaccine. 31(1): 135-140.

9 King K.W., Faulds D.H., Rosey E.L. \& Yancey R.J.Jr. 1997. Characterization of the gene encoding Mhp1 from Mycoplasma hyopneumoniae and examination of Mhp1's vaccine potential. Vaccine. 15(1): 25-35.

10 Maes D., Deluyker H., Verdonck M., Castryck F., Miry C., Vrijens B., Verbeke W., Viaene J. \& de Kruif A. 1999. Effect of vaccination against Mycoplasma hyopneumoniae in pig herds with an all-in/all-out production system. Vaccine. 17(9-10): 1024-1034.

11 Marchioro S.B., Maes D., Flahou B., Pasmans F., Del Pozo Sacristán R., Vranckx K., Melkebeek V., Cox E., Wuyts N. \& Haesebrouck F. 2013. Local and systemic immune responses in pigs intramuscularly injected with an inactivated Mycoplasma hyopneumoniae vaccine. Vaccine. 31(9): 1305-1311.

12 Marchioro S.B., Sácristan R.P., Michiels A., Haesebrouck F., Conceição F.R., Dellagostin O.A. \& Maes D. 2014. Immune responses of a chimaeric protein vaccine containing Mycoplasma hyopneumoniae antigens and LTB against experimental M. hyopneumoniae infection in pigs. Vaccine. 32(36): 4689-4694.

13 McGhee J.R., Mestecky J., Dertzbaugh M.T., Eldridge J.H., Hirasawa M. \& Kiyono H. 1992. The mucosal immune system: from fundamental concepts to vaccine development. Vaccine. 10(2): 75-88.

14 Morrison R.B., Hilley H.D. \& Leman A.D. 1985. Comparison of methods for assessing the prevalence and extent of pneumonia in market weight swine. Canadian Journal of Veterinary Research. 26(12): 381-384. 
15 Neutra M.R. \& Kozlowski P.A. 2006. Mucosal vaccines: the promise and the challenge. Nature Reviews Immunology. 6(2): 148-158.

16 Nicolet J., Paroz P. \& Bruggmann S. 1980. Tween 20 soluble proteins of Mycoplasma hyopneumoniae as antigen for an enzyme linked immunosorbent assay. Research in Veterinary Science. 29(3): 305-309.

17 Ogawa Y., Oishi E., Muneta Y., Sano A., Hikono H., Shibahara T., Yagi Y. \& Shimoji Y. 2009. Oral vaccination against mycoplasmal pneumonia of swine using a live Erysipelothrix rhusiopathiae vaccine strain as a vector. Vaccine. 27(33): 4543-4550.

18 Okamba F.R., Moreau E., Bouh K.C., Gagnon C.A., Massie B. \& Arella M. 2007. Immune responses induced by replication-defective adenovirus expressing the C-terminal portion of the Mycoplasma hyopneumoniae P97 adhesin. Clinical and Vaccine Immunology. 14(6): 767-774.

19 Okamba F.R., Arella M., Music N., Jia J.J., Gottschalk M. \& Gagnon C.A. 2010. Potential use of a recombinant replication-defective adenovirus vector carrying the $\mathrm{C}$-terminal portion of the $\mathrm{P} 97$ adhesin protein as a vaccine against Mycoplasma hyopneumoniae in swine. Vaccine. 28(30): 4802-4809.

20 Opriessnig T., Thacker E.L., Yu S., Fenaux M., Meng X.J. \& Halbur P.G. 2004. Experimental reproduction of postweaning multisystemic wasting syndrome in pigs by dual infection with Mycoplasma hyopneumoniae and porcine Circovirus type 2. Veterinary Pathology. 41(6): 624-640.

21 Simecka J.W., Davis J.K., Davidson M.K., Ross S.E., Stadtlander C.T.K.H. \& Cassell G.H. 1992. Mycoplasma diseases of animals. In: Maniloff J., McElhaney R.N., Finch L.R. \& Baseman J. B. (Ed.). Mycoplasmas: molecular biology and pathogenesis. American Society for Microbiology, Washington, pp.391-416.

22 Simionatto S., Marchioro S.B., Galli V., Brum C.B., Klein C.S., Rebelatto R., Silva E.F., Borsuk S., Conceição F.R. \& Dellagostin O.A. 2012. Immunological characterization of Mycoplasma hyopneumoniae recombinant proteins. Comparative Immunology, Microbiology \& Infectious Diseases. 35(2): 209-216.

23 Thacker E.L., Thacker B.J., Boettcher T.B. \& Jayappa H. 1998. Comparison of antibody production, lymphocyte stimulation and production induced by four commercial Mycoplasma hyopneumoniae bacterins. Journal of Swine Health and Production. 6(3): 107-112.

24 Thacker E.L., Thacker B.J., Kuhn, M., Hawkins P.A. \& Waters W.R. 2000. Evaluation of local and systemic immune responses induced by intramuscular injection of a Mycoplasma hyopneumoniae bacterin to pigs. American Journal of Veterinary Research. 61(11): 1384-1389.

25 Thacker E.L. 2006. Mycoplasmal Diseases. In: Straw B.E., Zimmerman J.J., D’ Allaire S. \& Taylor D.J. (Eds). Diseases of Swine. 9th edn. Ames: Blackwell Publishing, pp.701-717.

26 Villarreal I., Meyns T., Dewulf J., Vranckx K., Calus D., Pasmans F., Haesebrouck F. \& Maes D. 2011. The effect of vaccination on the transmission of Mycoplasma hyopneumoniae in pigs under field conditions. The Veterinary Journal. 188(1): 48-52.

27 Weltzin R., Guy B., Thomas W.D., Giannasca P.J. \& Monath T.P. 2000. Parenteral adjuvant activities of Escherichia coli heat-labile toxin and its B subunit for immunization of mice against Helicobacter pylori infection. Infection and Immunity. 68(5): 2775-2782.

28 Wilton J.L., Scarman A.L., Walker M.J. \& Djordjevic S.P. 1998. Reiterated repeat region variability in the ciliary adhesin gene of Mycoplasma hyopneumoniae. Microbiology. 144(7): 1931-1943.

29 Xiong Q., Wei Y., Xie H., Feng Z., Gan Y., Wang C., Liu M., Bai F., Xie F. \& Shao G. 2014. Effect of different adjuvant formulations on the immunogenicity and protective effect of a live Mycoplasma hyopneumoniae vaccine after intramuscular inoculation. Vaccine. 32(27): 3445-3451. 